\title{
Fructose-2,6-Bisphosphate synthesis by 6-Phosphofructo-2- Kinase/Fructose-2,6-Bisphosphatase 4 (PFKFB4) is required for the glycolytic response to hypoxia and tumor growth
}

\author{
Jason Chesney ${ }^{1, *}$, Jennifer Clark ${ }^{1, *}$, Alden C. Klarer ${ }^{1}$, Yoannis Imbert-Fernandez ${ }^{1}$, \\ Andrew N. Lane ${ }^{2}$ and Sucheta Telang ${ }^{1}$ \\ 1 James Graham Brown Cancer Center, Departments of Medicine (Hematology/Oncology), Pediatrics and Biochemistry and \\ Molecular Biology, University of Louisville, Louisville, KY \\ ${ }^{2}$ Current address: Markey Cancer Center, University of Kentucky, Lexington, KY \\ * These authors contributed equally to this work
}

Correspondence to: Sucheta Telang, email: sucheta.telang@lovisville.edu

Keywords: Glycolysis, 6-Phosphofructo-2-Kinase, Fructose-2,6-Bisphosphatase, Prostate Cancer, Lung Cancer

Received: July 03, 2014 Accepted: July 11, 2014 Published: July 13, 2014

This is an open-access article distributed under the terms of the Creative Commons Attribution License, which permits unrestricted use, distribution, and reproduction in any medium, provided the original author and source are credited.

\section{ABSTRACT}

Fructose-2,6-bisphosphate (F2,6BP) is a shunt product of glycolysis that allosterically activates 6-phosphofructo-1-kinase (PFK-1) resulting in increased glucose uptake and glycolytic flux to lactate. The F2,6BP concentration is dictated by four bifunctional 6-phosphofructo-2-kinase/fructose-2,6-bisphosphatases (PFKFB1-4) with distinct kinase:phosphatase activities. PFKFB4 is over-expressed in human cancers, induced by hypoxia and required for survival and growth of several cancer cell lines. Although PFKFB4 appears to be a rational target for anti-neoplastic drug development, it is not clear whether its kinase or phosphatase activity is required for cancer cell survival. In this study, we demonstrate that recombinant human PFKFB4 kinase activity is 4.3-fold greater than its phosphatase activity, siRNA and genomic deletion of PFKFB4 decrease F2,6BP, PFKFB4 over-expression increases F2,6BP and selective PFKFB4 inhibition in vivo markedly reduces F2,6BP, glucose uptake and ATP. Last, we find that PFKFB4 is required for cancer cell survival during the metabolic response to hypoxia, presumably to enable glycolytic production of ATP when the electron transport chain is not fully operational. Taken together, our data indicate that the PFKFB4 expressed in multiple transformed cells and tumors functions to synthesize F2,6BP. We predict that pharmacological disruption of the PFKFB4 kinase domain may have clinical utility for the treatment of human cancers.

\section{INTRODUCTION}

Glucose metabolism is regulated by a family of four bifunctional 6-phosphofructo-2-kinase/ fructose2,6-bisphosphatases (PFKFB1-4) that determine the concentration of fructose 2,6-bisphosphate (F2,6BP), which is a potent allosteric activator of the glycolytic enzyme 6-phosphofructo-1-kinase (PFK-1) [1]. Whereas F2,6BP activates PFK-1, several metabolic products including ATP, $\mathrm{H}+$ ions and citrate, allosterically inhibit PFK-1, indicating that PFK-1 is an essential metabolic sensor in the glycolytic pathway [2]. Given the central role that the PFKFB family members play in regulating glucose metabolism via PFK-1, understanding their specific functions within cells and tissues is of paramount importance to the study of a multitude of disease states caused by inflammation, ischemia and neoplastic transformation.

Within five years of the initial discovery of F2,6BP in 1980 [3, 4], multiple PFKFB enzyme activities were detected in several organs that were distinguished by having markedly reduced but still detectable fructose2,6-bisphosphatase activities relative to the liver PFKFB1 family member which has a near 1:1 kinase:bisphosphatase ratio (1). Interestingly, a distinct PFKFB activity was detected in the testes [5] and a human testes-specific 
PFKFB4 gene then was cloned in 1999 [6] suggesting that the unique metabolic requirements of sperm may necessitate a divergent PFKFB family member. Although several reports indicated that the testes PFKFB4 from various species display approximately 3-5 fold higher kinase than phosphatase activity $[5,7,8]$, suggesting that its function was predominantly to increase glycolytic flux into the 3-carbon portion of the pathway, one study of purified protein reported a near 1:1 ratio similar to that of the liver PFKFB1 family member [9].

In the early $2000 \mathrm{~s}$, Minchenko et al. demonstrated that the testes PFKFB4 was induced by hypoxia in multiple cancer cell lines and over-expressed in matched human lung, breast and colon tumor tissues relative to normal tissues from the same patients [10-13]. The functional requirement of PFKFB4 for tumor growth then was published in 2010 when researchers demonstrated that selective inhibition of PFKFB4 with siRNA suppressed the growth of human lung adenocarcinoma xenografts in athymic mice (U.S. Patent \#8,283,332; PFKFB4 Inhibitors and Methods of Using the Same). The importance of PFKFB4 as a potential target for the development of cancer therapeutics was then significantly expanded in 2012 by two independent groups that conducted unbiased screens for genes essential for cancer survival and found that PFKFB4 is required for both glioma stem-like cell [14] and prostate cancer cell survival [15] but not for normal cell survival. Taken together, these studies indicated that PFKFB4 may be a useful molecular target for the development of anti-neoplastic agents.

An essential first step in developing anti-cancer agents that inhibit PFKFB4 is to determine whether the kinase or phosphatase domain of this bifunctional enzyme are active in neoplastic cells. Whereas the kinase activity will produce F2,6BP and increase flux down the glycolytic pathway producing ATP, the phosphatase activity will decrease F2,6BP, reduce PFK-1 activity and increase the oxidative pentose shunt activity and NADPH via the availability of glucose 6-phosphate which is in equilibrium with fructose 6-phosphate (F6P) through glucose 6-phosphate isomerase. Given that the intracellular concentration of the substrate of the kinase domain (F6P) is $>10,000$ fold the substrate of the phosphatase domain (F2,6BP) in transformed cells (e.g. MCF-7 cells: F6P, $50 \pm 24 \mathrm{nmol} / \mathrm{mg}$ protein [16]; F2,6BP, 2.97 $\pm .21 \mathrm{pmol} /$ $\mathrm{mg}$ protein [17]), we predicted that the kinase activity of PFKFB4 would be the dominant function of this enzyme in transformed cells.

In the present study, we find that PFKFB4 functions primarily to produce $\mathrm{F} 2,6 \mathrm{BP}$, thus stimulating glycolytic flux to lactate and the Kreb's cycle in order to generate ATP and anabolic precursors. Accordingly, the synthesis of small molecule inhibitors of the PFKFB4 kinase domain is anticipated to enable the development of novel agents for the treatment of cancer.

\section{RESULTS}

\section{High Expression of PFKFB4 in Several Normal Tissues and in Lung Adenocarcinomas}

We first conducted a simultaneous analysis of PFKFB1-4 mRNA expression in normal human tissues using real-time RT-PCR for each family member. We observed high expression of: PFKFB1 in liver and skeletal muscle; PFKFB2 in heart, lung, skeletal muscle, kidney, pancreas and testes; and PFKFB4 in placenta, lung, skeletal muscle, pancreas, spleen, prostate, testes, ovary, colon and leukocytes (Fig. 1A). PFKFB3, which has been characterized as being inducible [18] and ubiquitous [19], was expressed at very low levels in all tissues with the exception of leukocytes (Fig 1A). We then used multiplex RT-PCR and real-time RT-PCR to assess PFKFB1-4 mRNA expression in normal lung tissues and adjacent lung adenocarcinomas from five patients. Only PFKFB4 mRNA was over-expressed in each of these patients' tumors (Fig. 1B, C). Further examination of a larger cohort of lung adenocarcinomas and adjacent normal tissues from 18 patients by immunohistochemical analyses revealed high PFKFB4 protein expression relative to normal lung alveoli (Fig. 1D, E).

\section{The Kinase:Phosphatase Ratio of PFKFB4 is 4.3:1 and Selective Inhibition of PFKFB4 Expression Reduces F2,6BP in Multiple Cancer Cell Lines}

We expressed and purified recombinant human PFKFB4 and compared the kinase and phosphatase activities to those of the inducible PFKFB3 recombinant protein. Given the recent reports that PFKFB4 functions as a dominant bisphosphatase in cancer cells [15], we were surprised to find that recombinant human PFKFB4 had significant kinase activity that was $\sim 15 \%$ that of the PFKFB3 family member whose kinase domain has previously been established to set the intracellular concentration of F2,6BP in multiple cell lines (PFKFB4 kinase $\mathrm{V}_{\max }=5.06 \pm 0.2 \mathrm{mU} / \mathrm{mg}$, PFKFB3 kinase $\mathrm{V}_{\max }=$ $38.4 \pm 2.3 \mathrm{mU} / \mathrm{mg}$ ) (Fig. 2A) [18, 20, 21]. In contrast, both PFKFB4 and PFKFB3 were found to have relatively low phosphatase activities (PFKFB4 bisphosphatase $\mathrm{V}_{\max }=1.17 \pm 0.02 \mathrm{mU} / \mathrm{mg}$; PFKFB3 bisphosphatase $\mathrm{V}_{\max }$ $=0.47 \pm 0.035 \mathrm{mU} / \mathrm{mg}$ ) and the kinase:bisphosphatase ratios for PFKFB4 and PFKFB3 were calculated to be 4.3:1 and 81.7:1, respectively (Fig. 2A). Since the concentration of the kinase substrate, F6P, is so much higher than that of the bisphosphatase substrate, F2,6BP (e.g. $\mathrm{H} 460$ cell $[\mathrm{F} 6 \mathrm{P}]=12518 \pm 1104 \mathrm{pmol} / \mathrm{mg}$ protein; $[\mathrm{F} 2,6 \mathrm{BP}]=4.47 \pm .21 \mathrm{pmol} / \mathrm{mg}$ protein) and because the kinase:phosphatase is $4.3: 1$, we predicted that selective inhibition of PFKFB4 would preferentially inhibit the 
kinase function of this bifunctional enzyme and thus result in a reduction in $\mathrm{F} 2,6 \mathrm{BP}$. We found that this did indeed occur in six out of seven transformed cancer cell lines derived from lung, colon, prostate and breast that were each transfected with two separate PFKFB4-specific siRNAs (Fig. 2B). Importantly, genomic deletion of $P f k f b 4$ in LT-immortalized fibroblasts also caused a marked reduction in F2,6BP (Fig. 2C). Since PFKFB3, unlike PFKFB4, has been established to function as a kinase in transformed cells, we next examined the effect of PFKFB3 siRNA transfection on three transformed cell lines and similarly observed a marked reduction in the steady-state concentration of F2,6BP (Fig. 2D).

\section{Selective Inhibition of PFKFB4 Expression Reduces Glycolysis and ATP in Multiple Cancer Cell Lines}

We examined the effect of the two PFKFB4 siRNA molecules and genomic deletion of $P f k f b 4$ on glycolysis (measured by the production of ${ }^{3} \mathrm{H}_{2} \mathrm{O}$ from $\left[5-{ }^{3} \mathrm{H}\right]$ glucose), ATP and NADPH. We predicted that if the kinase function of PFKFB4 is dominant, then PFKFB4 inhibition would suppress (rather than increase) glycolysis and decrease the concentration of ATP which is produced downstream of PFK-1. We found that both glycolysis and ATP were reduced in all five cell lines examined although the ATP
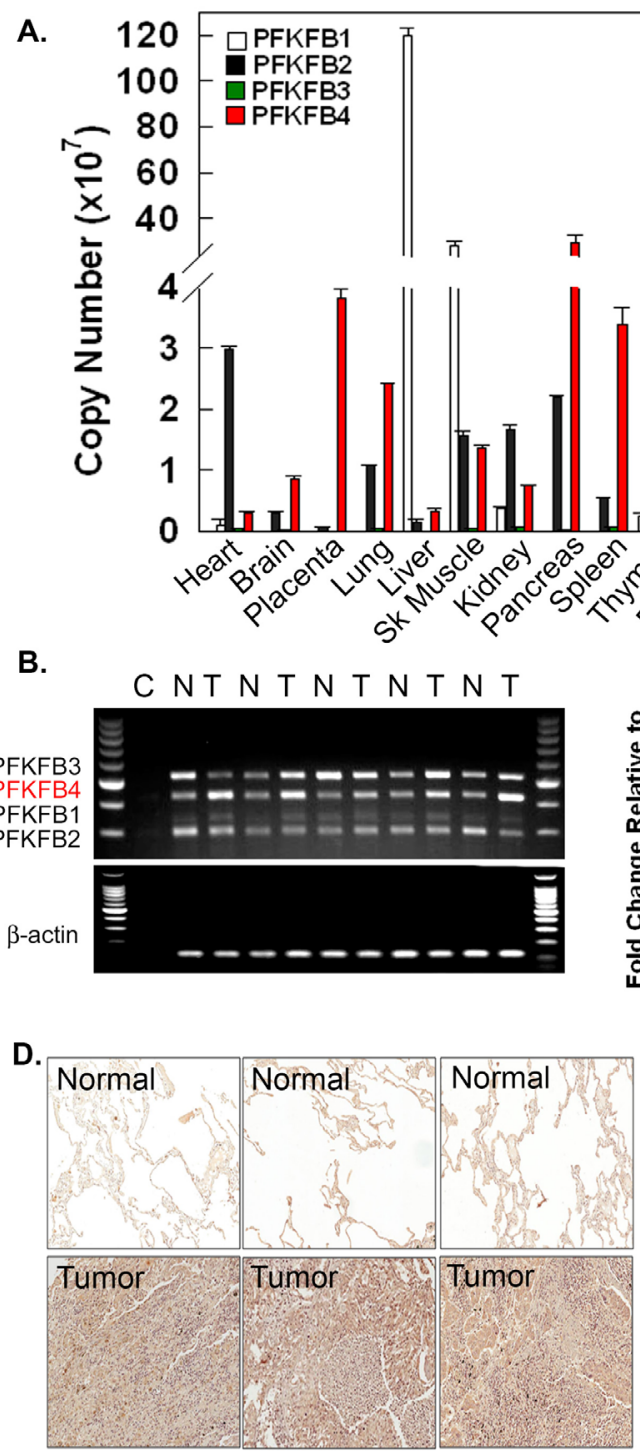

C.

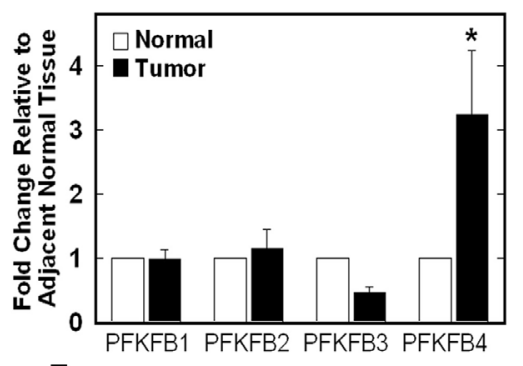

E.

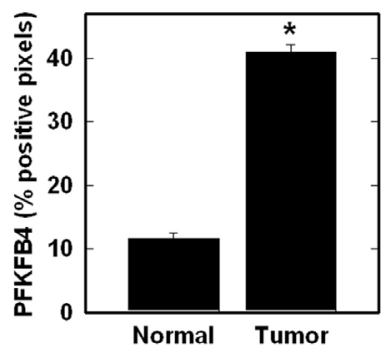

Figure 1: PFKFB4 Is Expressed in Multiple Normal Tissues and Over-Expressed in Human Lung Adenocarcinomas. PFKFB1-4 mRNA expression in multiple normal tissues was assessed by real-time RT-PCR and expressed by copy number (A). Matched normal $(\mathrm{N})$ and lung adenocarcinoma $(\mathrm{T})$ tissue were analyzed by multiplex RT-PCR (B) and by real-time RT-PCR expressed as fold change relative to adjacent normal tissues (C). Immunohistochemical staining of lung adenocarcinomas and normal lung was conducted on patients' biopsies, three representative sections are shown (D) and positive pixels were quantitated (E). Data are expressed as the mean \pm $\mathrm{SD}$ of three experiments. ${ }^{*} p$ value $<0.001$ compared to normal tissue controls. 
A.
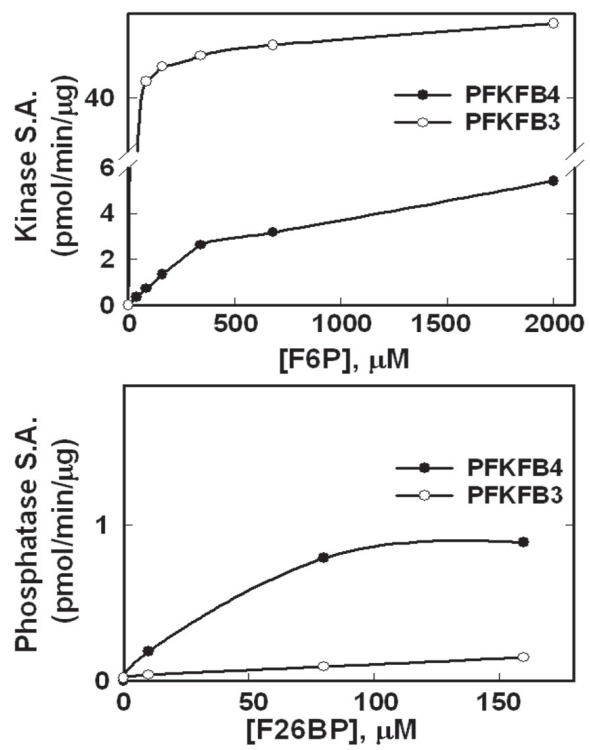

C.
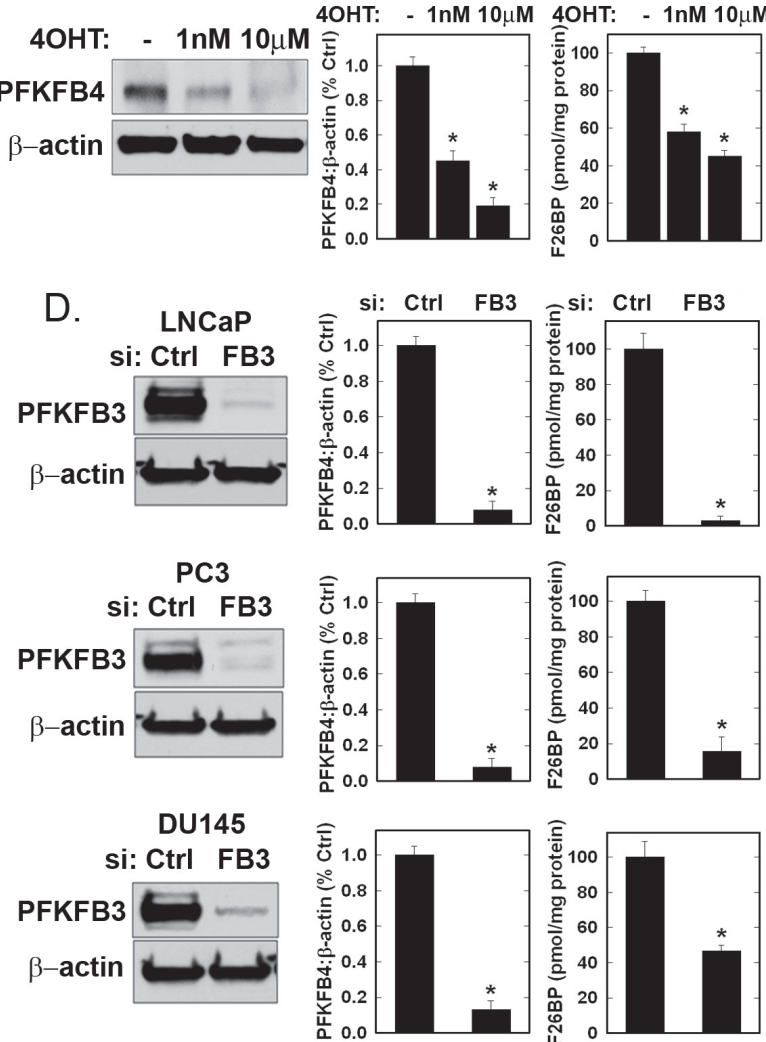

B.
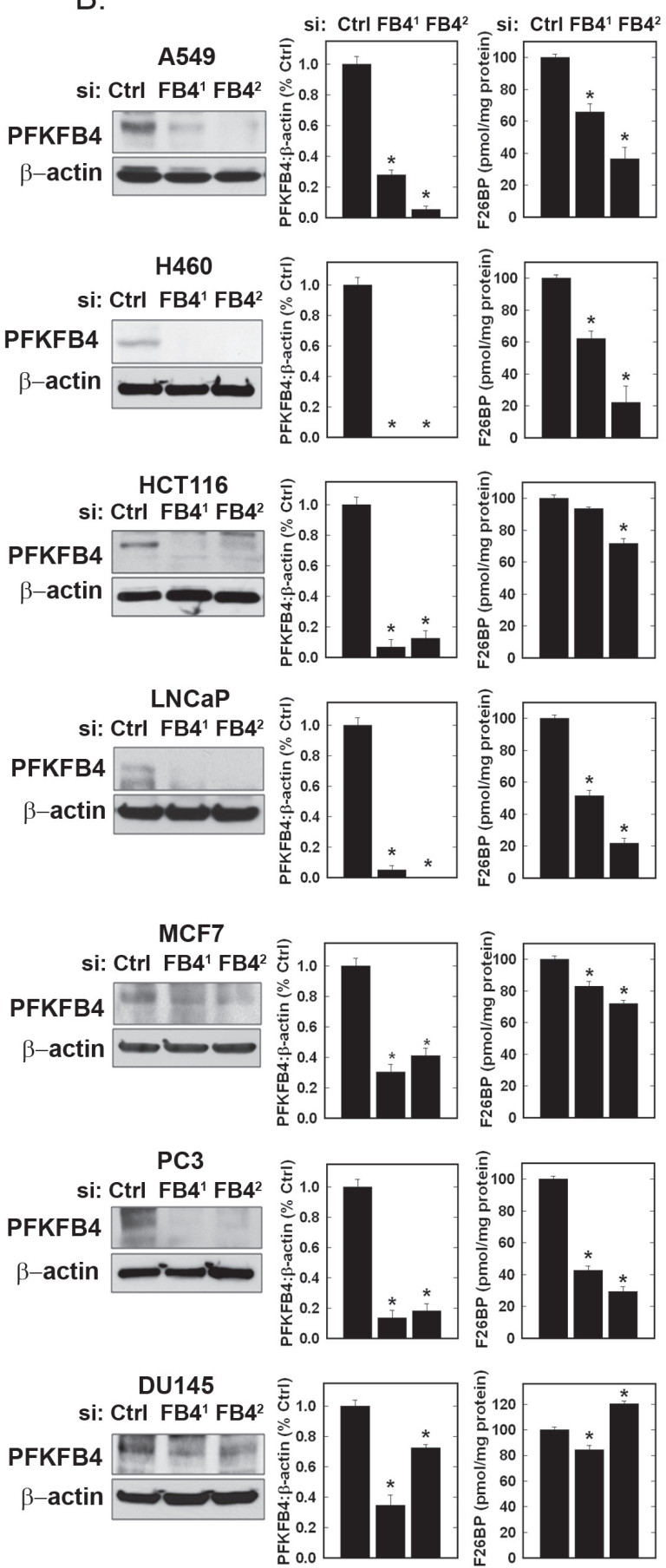

Figure 2: Recombinant PFKFB3 and PFKFB4 Kinase and Phosphatase Activities and Effects of PFKFB3 and PFKFB4 Inhibition on Intracellular F2,6BP in Cancer Cells. Human PFKFB3 and PFKFB4 were expressed and relative kinase and phosphatase specific activities (S.A.) were determined (A). The indicated cancer cell lines were transfected with control siRNA (Ctrl) or two PFKFB4 siRNAs (FB4 ${ }^{1}$ and FB4 ${ }^{2}$ ) and, after 48 hours, protein expression examined by Western blot and quantitated by densitometry and intracellular F2,6BP was measured (B). Large T antigen-immortalized, tamoxifen (4OHT)-inducible $P f k f b 4^{-/}$lung fibroblasts were exposed to $1 \mathrm{nM}$ and $10 \mu \mathrm{M} 4 \mathrm{OHT}$ (ethanol used as vehicle control) and analyzed for protein expression and F2,6BP after 48 hours (C). The indicated cancer cell lines were transfected with an established PFKFB3 siRNA and analyzed for protein expression and F2,6BP 48 hours later (D). Data are expressed as the mean $\pm \mathrm{SD}$ of three experiments. ${ }^{*} p$ value $<0.001$ compared to vehicle or control siRNA. 
depletion in MCF-7 cells was not statistically significant (Fig. 3A). Although we observed variable effects on NADPH, we did find that PFKFB4 inhibition increased NADPH in H460, A549, LNCaP and MCF-7 cells, presumably as a result of increased availability of F6P for the activity of glucose 6-phosphate isomerase and the oxidative pentose shunt (Fig. 3A). Importantly, we found that $P f k f b 4$ genomic deletion reduced glycolysis and ATP and increased NADPH, all findings consistent with a requirement of PFKFB4 for the activation of PFK-1 (Fig. 3B).

\section{Over-Expression of PFKFB4 Increases F2,6BP, Glycolysis and ATP and reduces NADPH}

transfection and genomic deletion both reduced F2,6BP, we predicted that over-expression would have the opposite effect on F2,6BP and subsequent metabolic events. We found that PFKFB4 over-expression increased F2,6BP, glycolysis and ATP, and decreased NADPH in each of the examined transformed cell lines (Fig. 4). We also examined the effect of addition of $5 \mathrm{mM}$ pyruvate on the depletion of ATP caused by PFKFB4 siRNA in H460 cells and found that pyruvate partially reversed the ATP depletion, further supporting a role for PFKFB4 in increasing the glycolytic production of ATP (ATP, pmoles/ $\mu \mathrm{g}$ protein: Ctrl siRNA, 39.9 \pm 0.2 ; PFKFB4 siRNA 9.94 \pm 0.5 ; Ctrl siRNA + Pyruvate, $40.4 \pm 0.13$; PFKFB4 siRNA + pyruvate, $33.1 \pm 1.1 ; p$ value $<0.01)$.

Given that the kinase activity of PFKFB4 is 4.3fold the phosphatase activity and that PFKFB4 siRNA

A.
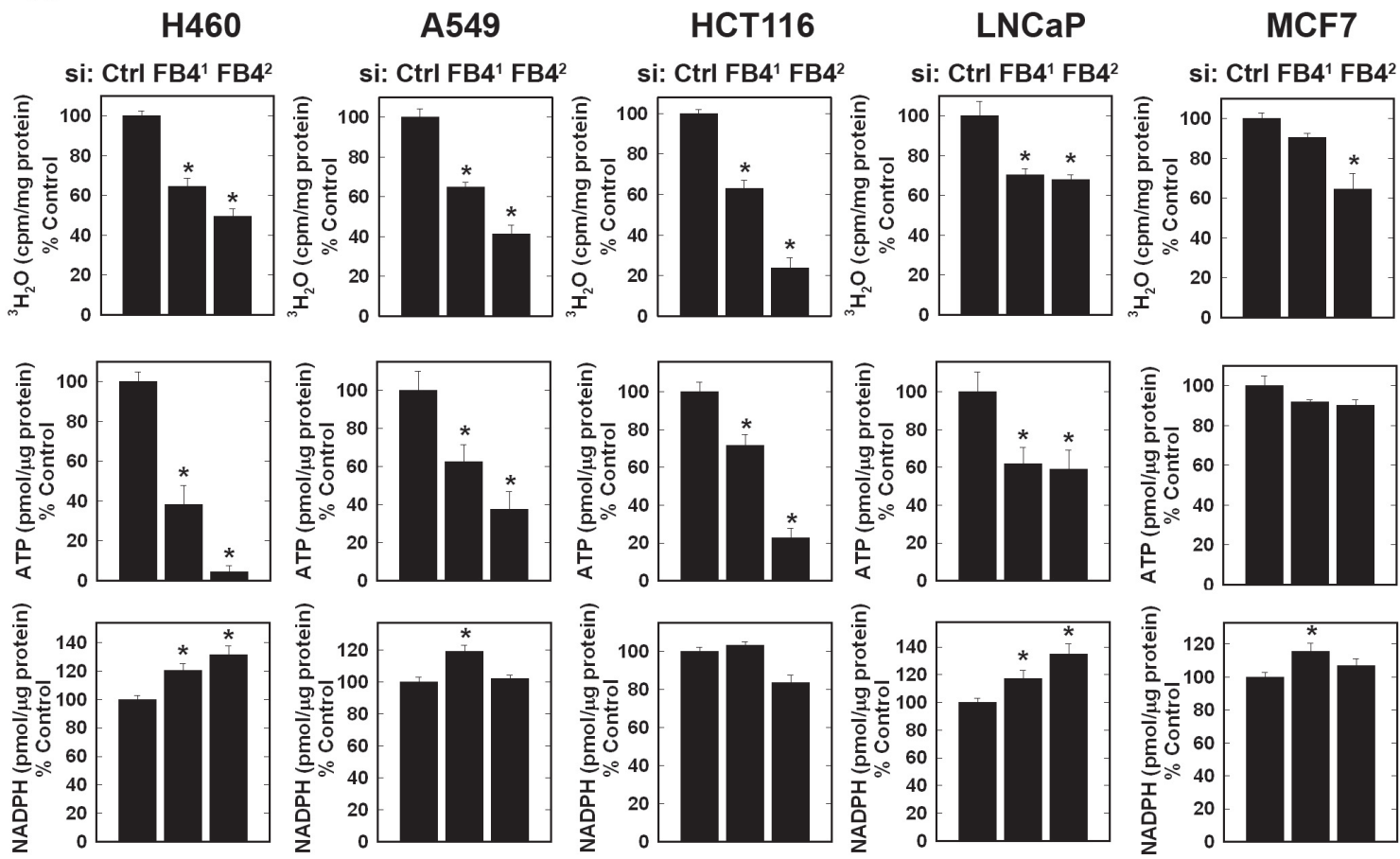

\section{B. PFKFB4-1-}
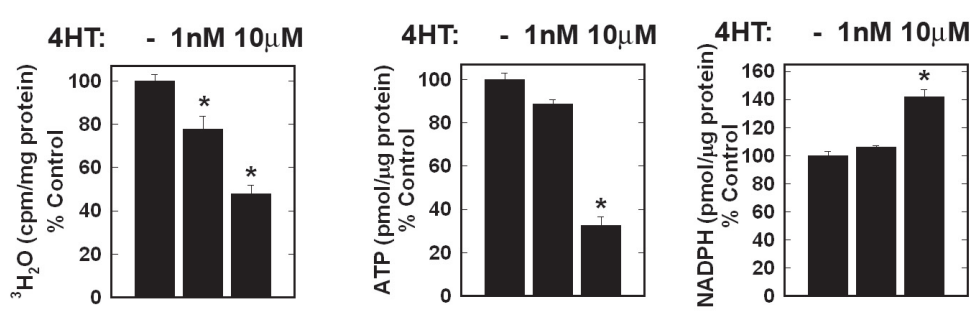

Figure 3: Effects of PFKFB4 Inhibition on Cancer Cell Glycolysis, ATP and NADPH. The indicated cell lines were transfected with control siRNA (Ctrl) or two PFKFB4 siRNAs (FB4 ${ }^{1}$ and $\left.\mathrm{FB} 4{ }^{2}\right)$ and glycolysis $\left({ }^{3} \mathrm{H}_{2} \mathrm{O}\right.$ production from $\left[5-{ }^{3} \mathrm{H}\right]$ glucose), intracellular ATP and NADPH were measured (A). Large T antigen-immortalized, tamoxifen (4OHT)-inducible Pfkfb $4^{-/}$lung fibroblasts were exposed to $1 \mathrm{nM}$ and $10 \mu \mathrm{M} 4 \mathrm{OHT}$ and analyzed for glycolysis, ATP and NADPH 48 hours later (B). Data are expressed as the mean \pm SD of three experiments. $* p$ value $<0.01$ compared to vehicle or control siRNA. 


\section{Inhibition of PFKFB4 Suppresses H460 Lung Adenocarcinoma F2,6BP, Glucose Uptake and Growth In Vivo}

In order to determine if PFKFB4 functions to synthesize F2,6BP in vivo, we stably transfected $\mathrm{H} 460$ cells with PFKFB4 shRNA and confirmed reduced PFKFB4 protein expression in vitro (Fig 5A). We found that stable PFKFB4 inhibition reduced anchorageindependent growth as soft agar colonies and as tumors (Fig. 5A, B). We then examined resected tumors after two weeks of growth and observed a marked reduction in tumor F2,6BP and ATP and an increase in apoptotic cells in the tumors by immunohistochemistry for cleaved caspase 3 (Fig 5C, D, F) We also found a small increase in NADPH in the PFKFB4 shRNA tumors in comparison with control tumors which was not statistically significant (Fig 5E). In addition, we examined uptake of $\left[{ }^{18} \mathrm{~F}\right]$-FDG in PFKFB4 shRNA and control tumors by PET scan and observed reduced tumor $\left[{ }^{18} \mathrm{~F}\right]$-FDG uptake in the PFKFB4 shRNA tumors (Fig. 5G) as has also been described as a result of PFKFB3 inhibition. Taken together, these in vivo studies provide strong evidence that PFKFB4 supports tumor growth by functioning as a kinase to synthesize F2,6BP.
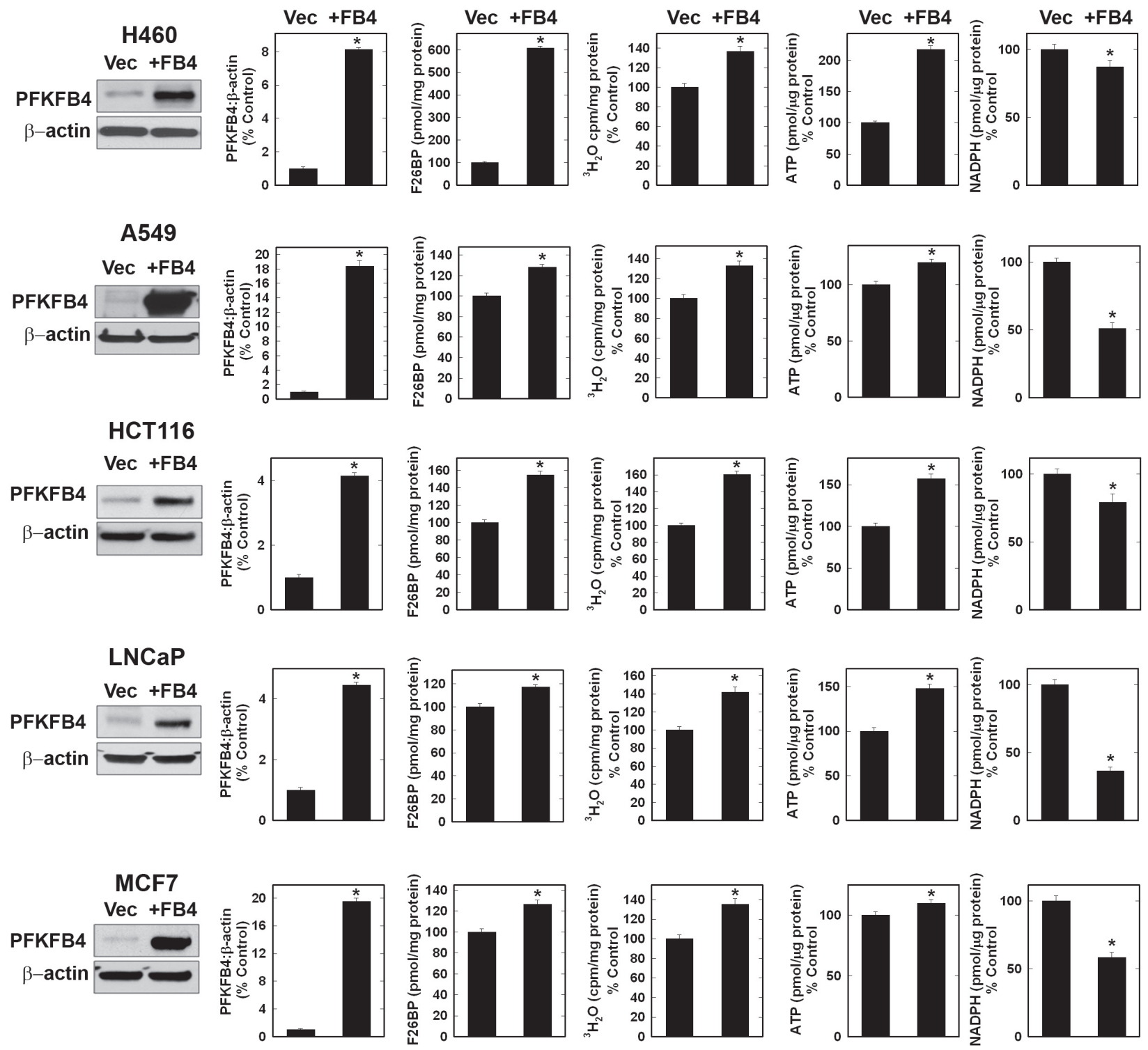

Figure 4: Over-Expression of PFKFB4 in Cancer Cells Increases F2,6BP, Glycolysis and ATP but Decreases NADPH. The indicated cancer cell lines were transfected with either empty pCMV-XL4 (Vec) or pCMV-XL4 containing full-length PFKFB4 (+FB4) and analyzed 48 hours later by Western blot and densitometry, and for F2,6BP, glycolysis, ATP and NADPH. Data are expressed as the mean $\pm \mathrm{SD}$ of three experiments. ${ }^{*} p$ value $<0.01$ compared to empty pCMV-XL4 vector. 
PFKFB4 mRNA and Protein Expression Are Increased by Hypoxia and Required for HypoxiaInduced F2,6BP, Glucose Uptake and Glycolysis

Both PFKFB3 and PFKFB4 mRNA previously have been found to be induced by hypoxia [10,12, 13, 22-24] and we confirmed these past observations in $\mathrm{H} 460$ cells (Fig. 6A). In order to compare the functional relevance of PFKFB3 and PFKFB4 under hypoxic conditions, we transfected H460 cells with PFKFB4 or PFKFB3 siRNA under $21 \%$ and $1 \%$ oxygen. We observed a reduction in the hypoxic induction of both enzyme mRNAs as a result of the siRNA molecules (Fig. 6A). Hypoxia strongly induced PFKFB4 protein expression, as has been observed in other cell lines (Fig. 6A-C). Interestingly, we found a far lower induction of PFKFB3 protein which may already be maximally expressed as a result of the multitude of genetic alterations that increase PFKFB3 protein expression such as PTEN loss and Ras activation (e.g. H460 cells express $\left.K R A S^{Q 61 H}\right)[20,25,26]$. Importantly, transfection with either PFKFB4 or PFKFB3 siRNA caused an attenuation
A.

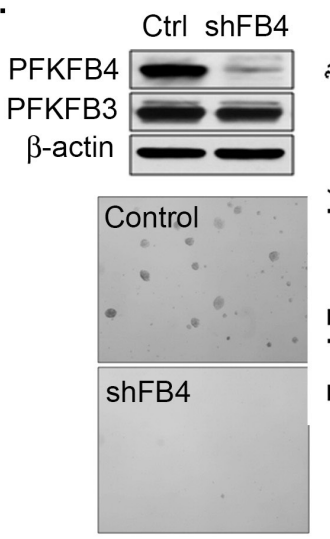

C.

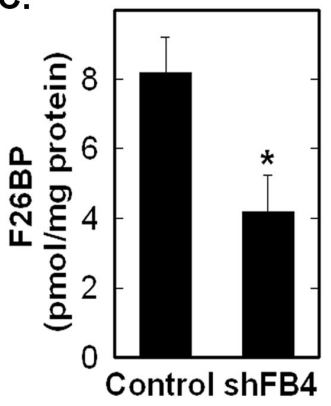

F.

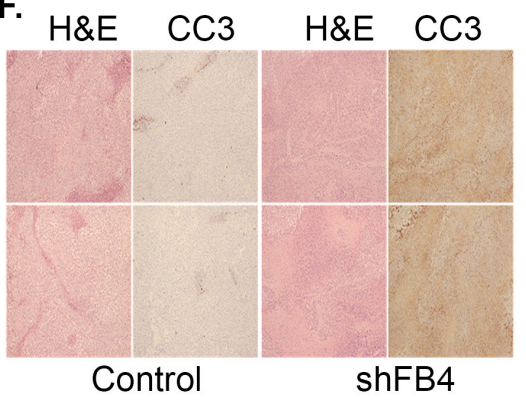

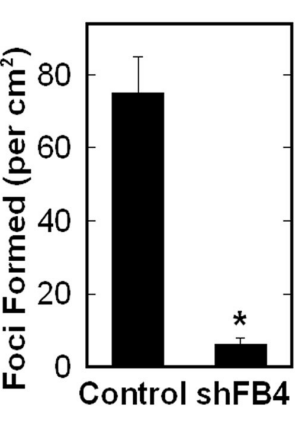

B.

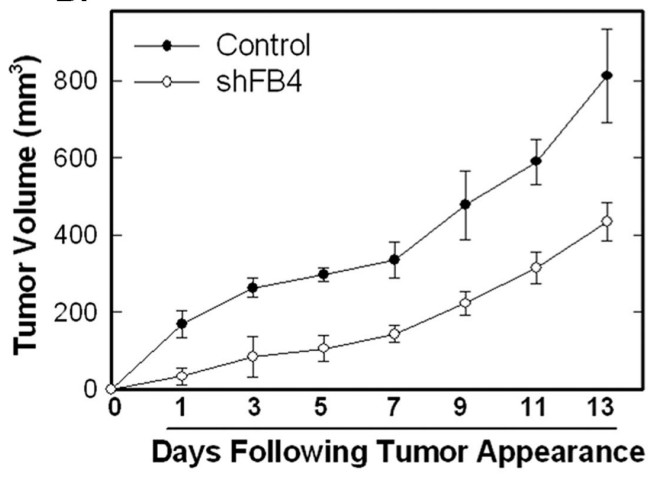

D.

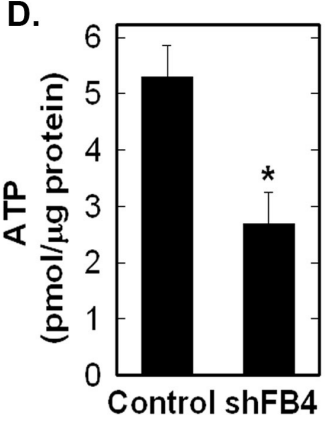

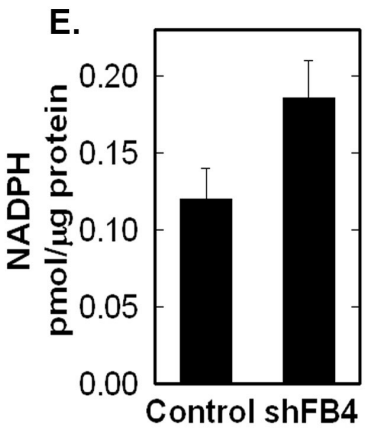

G.

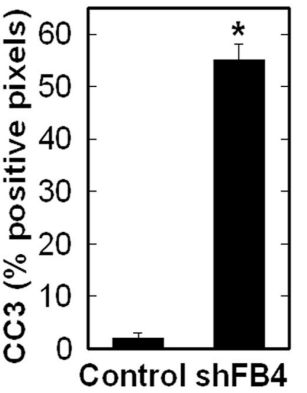

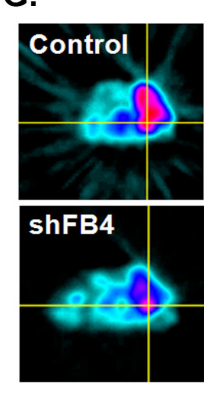

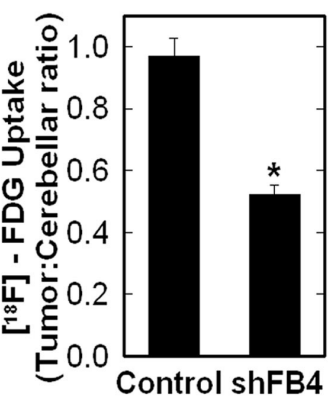

Figure 5: Stable shRNA Knock-Down of PFKFB4 Reduces Tumor Growth, Glucose Uptake and F2,6BP, and Increases Apoptosis. H460 cells were stably transduced with PFKFB4 (shFB4) or control (Control, Ctrl) shRNA and assessed for PFKFB4 and PFKFB3 protein expression by Western blot analysis (A), soft agar colony formation (A), tumor growth in athymic mice (B). F2,6BP concentration (C), ATP (D), and NADPH (E) were measured in tumors following resection. Data are expressed as the mean \pm SD of three experiments. Tumor sections were examined for apoptosis by cleaved caspase 3 (CC3) immunohistochemistry, with representative images on the left and positive pixels quantified on the right $(\mathrm{F})$. The data is depicted as $\%$ positive pixels/total pixels $\pm \mathrm{SD}$. Tumors in mice were examined for ${ }^{18} \mathrm{~F}$-FDG uptake in vivo by micro-PET imaging. Regions of interest in the tumors and cerebellum were quantified in quadruplicate (right) and representative transverse cuts are shown on the left (G). ${ }^{*} p$ value $<0.001$ compared to control shRNA. 
of F2,6BP, glucose uptake and glycolysis stimulated by hypoxia (Fig. 6D-F). The concentrations of both ATP and NADPH were decreased under hypoxia and PFKFB4 siRNA exacerbated the ATP decrease, indicating that PFKFB4 may be essential for the glycolytic response to hypoxia in these cells (Fig. 6G, H).

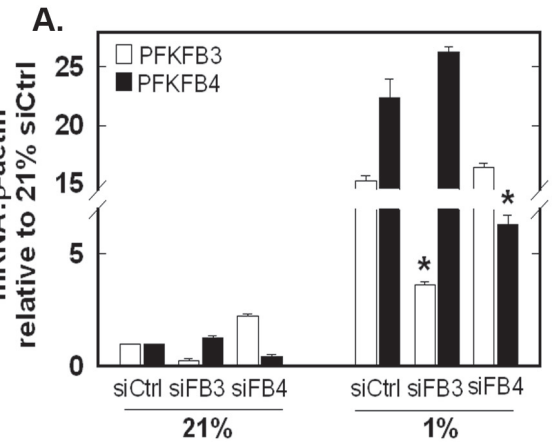

B.

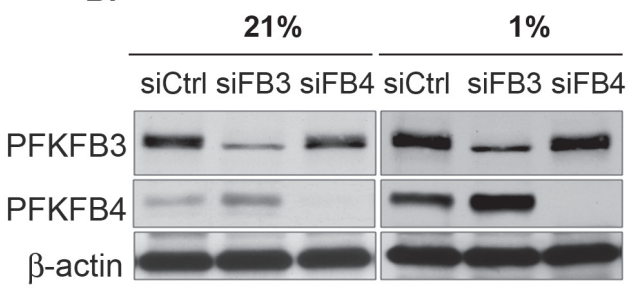

C.

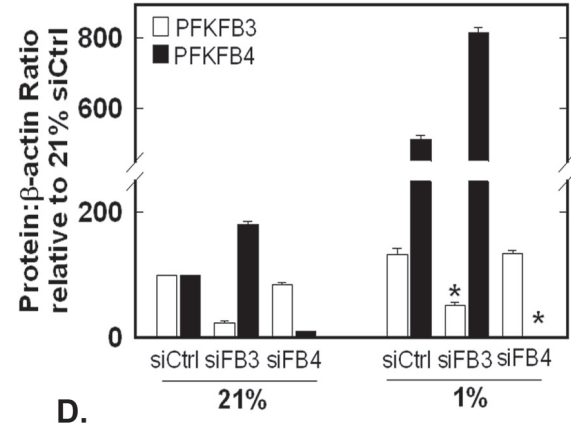

D.

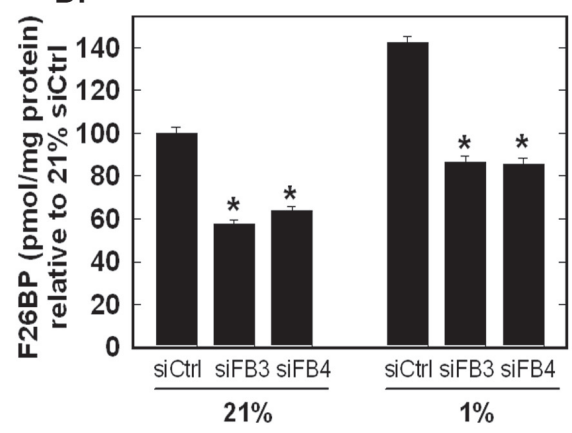

PFKFB3 or PFKFB4 Inhibition in $\mathbf{H 4 6 0}$ Cells Reduces Glycolytic Flux to Lactate and Glutamate

Given that both PFKFB3 and PFKFB4 siRNA suppressed hypoxia-induced F2,6BP, we suspected that inhibition of PFKFB3 or PFKFB4 would result in
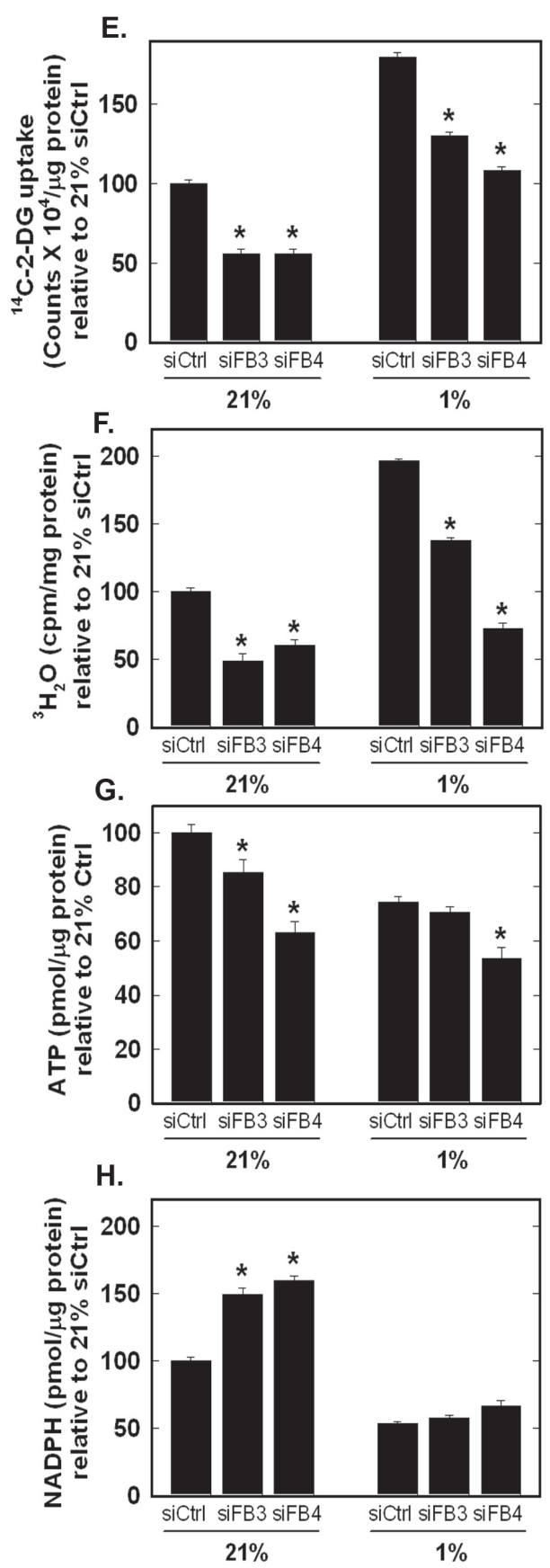

Figure 6: Role of PFKFB3 and PFKFB4 in Hypoxia-Induced F2,6BP production, Glucose Uptake, Glycolysis, ATP and NADPH. H460 cells were transfected with control siRNA (siCtrl), PFKFB3 siRNA (siFB3) or PFKFB4 siRNA (siFB4), cultured in $21 \%$ oxygen or $1 \%$ oxygen and examined for PFKFB3 and PFKFB4 mRNA expression (A) and protein expression by Western blot analysis and densitometry (B and C), F2,6BP (D), ${ }^{14} \mathrm{C}-2-\mathrm{DG}$ uptake (E), glycolysis $\left({ }^{3} \mathrm{H}_{2} \mathrm{O}\right.$ production from $\left.\left[5-{ }^{3} \mathrm{H}\right] \mathrm{glucose}\right)(\mathrm{F})$, intracellular ATP $(\mathrm{G})$ and NADPH $(\mathrm{H})$. Data are expressed as the mean $\pm \mathrm{SD}$ of three experiments. ${ }^{*} p$ value $<0.01$ compared to control siRNA. 
reduced glycolytic flux to lactate and into the TCA cycle product, glutamate, under hypoxic conditions. Whereas we found that both PFKFB3 and PFKFB4 siRNAs caused a reduction in the conversion of ${ }^{13} \mathrm{C}$-glucose to lactate and glutamate as assessed by NMR, the PFKFB4 siRNA caused a far greater reduction in the conversion to both products under both normoxic and hypoxic conditions (Fig. 7A, B).
PFKFB3 or PFKFB4 Inhibition in $\mathbf{H 4 6 0}$ Cells Increases Apoptosis Under Normoxic and Hypoxic Conditions

Maintenance of intracellular ATP is required to prevent apoptosis and we postulated that PFKFB3 or PFKFB4 inhibition would increase apoptosis. We observed a marked increase in apoptotic $\mathrm{PI}^{+} / \mathrm{Annnexin} \mathrm{V}^{+}$
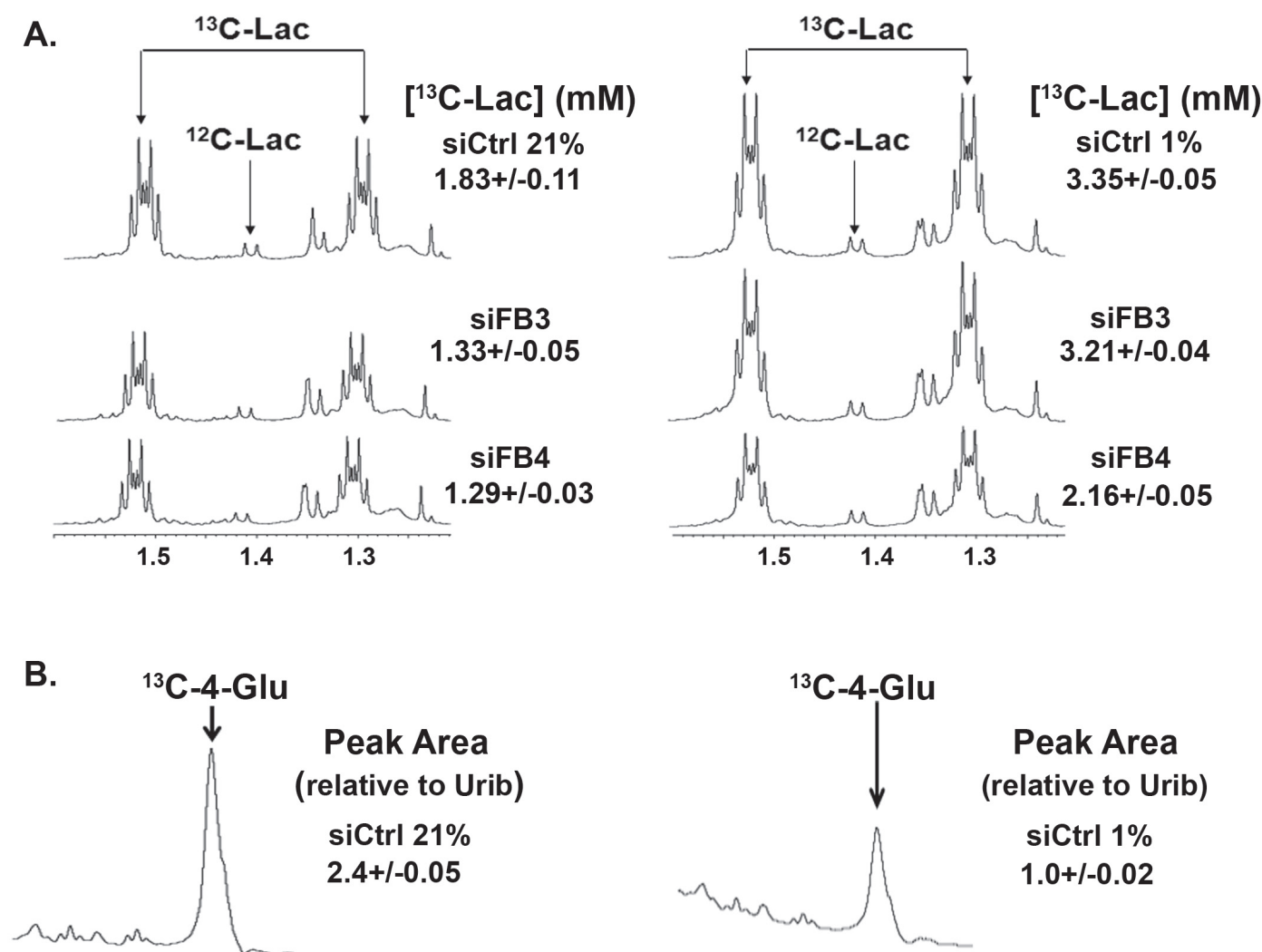

\author{
Peak Area \\ (relative to Urib)
}

siCtrl $1 \%$

$1.0+/-0.02$

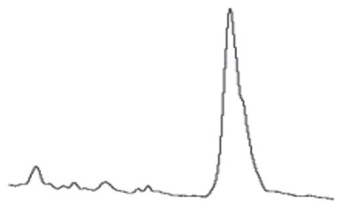

SiFB3 $21 \%$

$1.8+/-0.01$

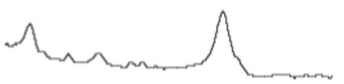

siFB3 $1 \%$

$0.53+/-0.04$

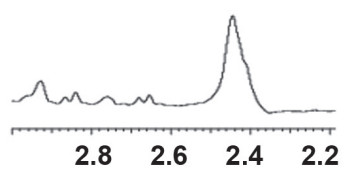

siFB4 21\%

$1.4+/-0.03$

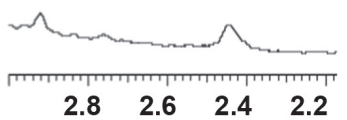

siFB4 $1 \%$

$0.22+/-0.02$

Figure 7: Role of PFKFB3 and PFKFB4 in the Conversion of ${ }^{13} \mathrm{C}$-Glucose into ${ }^{13} \mathrm{C}$-Lactate and ${ }^{13} \mathrm{C}-4-\mathrm{Glutamate}$. $\mathrm{H} 460$ cells were transfected with control siRNA (siCtrl), PFKFB3 siRNA (siFB3) or PFKFB4 siRNA (siFB4), cultured in 21\% oxygen or 1\% oxygen, pulsed with uniformly-labeled $1{ }^{3} \mathrm{C}$-glucose for hours and extracted for NMR analyses of ${ }^{13} \mathrm{C}$-lactate and ${ }^{13} \mathrm{C}$-4-glutamate. $(\mathrm{A})$ 1D NMR spectra of media extracts showing the region corresponding to the methyl groups of lactate and essential amino acids. Satellite peaks were integrated and normalized to internal DSS concentration to give the concentrations noted. Under these conditions, nearly all of the lactate derives from glucose. (B) $1 \mathrm{D}{ }^{1} \mathrm{H}\left\{{ }^{13} \mathrm{C}\right\}$ HSQC spectra of cellular extracts showing selective enrichment at the $\mathrm{C} 4$ atom of glutamate (Glu) under normoxia, $\left(21 \% \mathrm{O}_{2}\right.$, left) and under hypoxia $\left(1 \% \mathrm{O}_{2}\right.$, right). The $\mathrm{C} 4$ position of Glu becomes enriched by addition of glucose-derived acetate to oxaloacetate. The resulting ${ }^{13} \mathrm{C} 2$ citrate is converted by the TCA cycle to 2-oxoglutarate and transaminated to Glu, showing that the TCA cycle remains active in these cells, but is greatly reduced under $1 \%$ oxygen and by PFKFB3 and PFKFB4 knockdown. Values on the right denote ratios of peak areas normalized to uridine ribose (Urib) H1', which correspond to relative amounts of glucose-derived ${ }^{13} \mathrm{C}$. Numbers expressed are the mean $\pm \mathrm{SD}$ of three experiments. 
cells and cleaved PARP with inhibition of either family member under both normoxia and hypoxia but observed the greatest number of apoptotic cells as a result of the combination of hypoxia and PFKFB4 inhibition (Fig. 8AC).

\section{PFKFB4 Expression Correlates With Hypoxia in Human Lung Adenocarcinoma Xenografts}

We examined serial sections of human H460 lung adenocarcinoma xenograft tumors resected from athymic mice for expression of PFKFB4 and PFKFB3 and correlated the expression levels of these proteins using carbonic anhydrase IX, a potent transcriptional target of HIF- $1 \alpha$, as a hypoxia marker. We observed a statistically significant correlation between PFKFB4 and carbonic anhydrase IX expression but observed no correlation between PFKFB3 and carbonic anhydrase IX (Figure 9AD).

\section{DISCUSSION}

The main objective of this study was to determine which of the two enzymatic domains of PFKFB4 is

A.

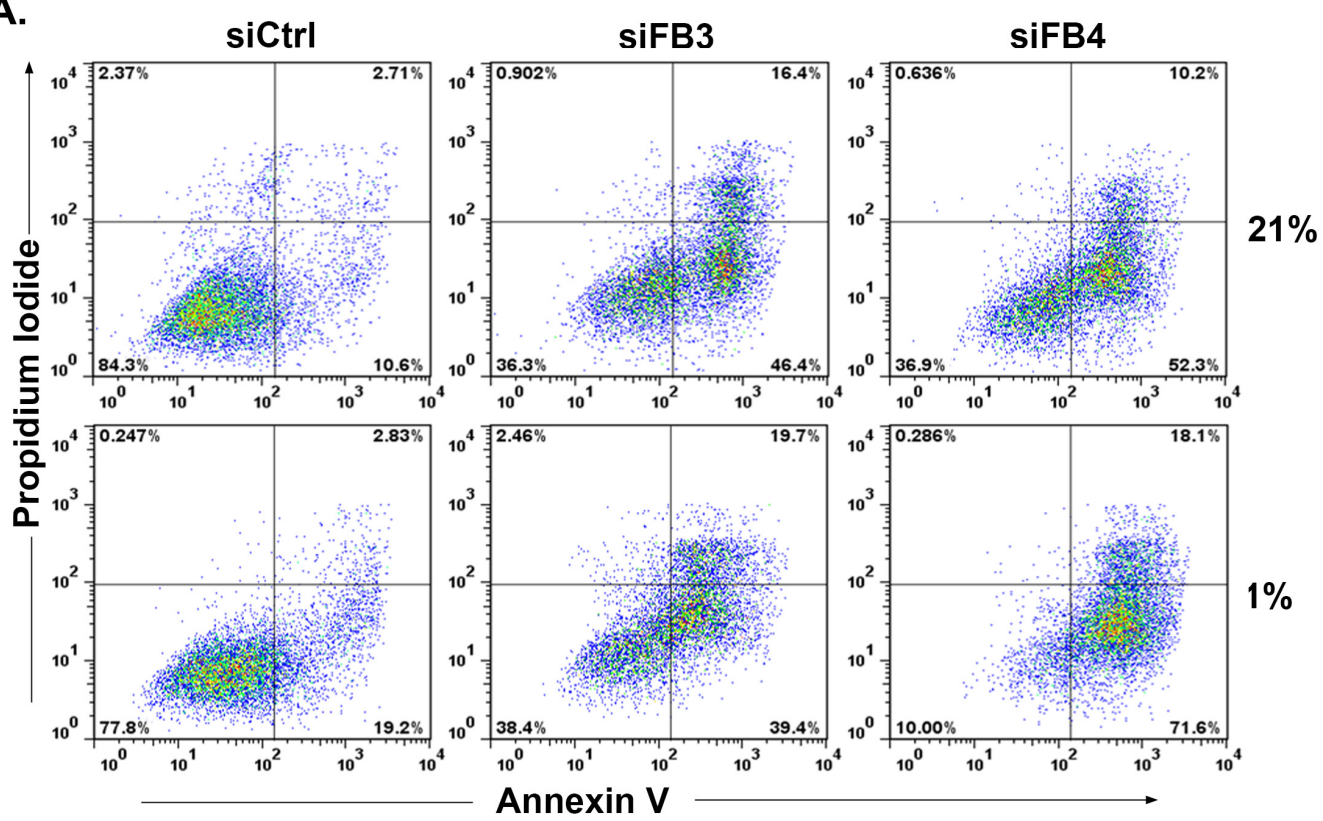

B.

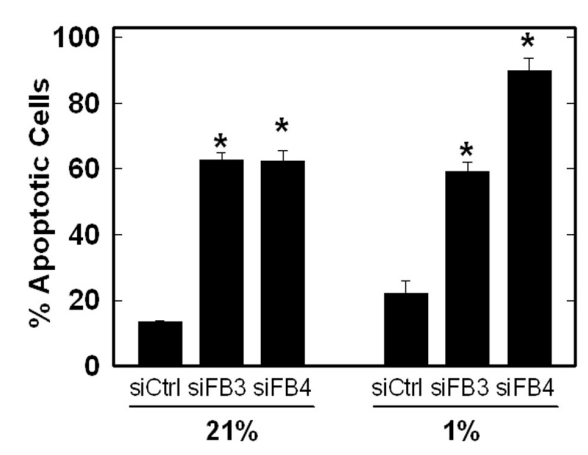

C.

$21 \%$

$1 \%$

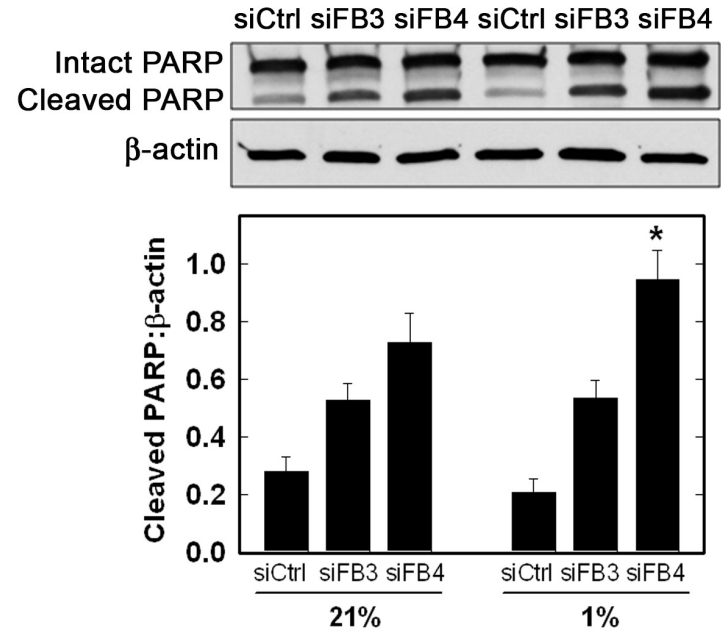

Figure 8: Effect of PFKFB3 or PFKFB4 siRNA Transfection on Apoptosis. H460 cells were transfected with control siRNA (siCtrl), PFKFB3 siRNA (siFB3) or PFKFB4 siRNA (siFB4), cultured in 21\% oxygen or 1\% oxygen for 48 hours, and analysed for apoptosis by flow cytometry of propidium iodide and annexin $\mathrm{V}$ positive cells (A and $\mathrm{B}, \mathrm{PI}^{+}+\mathrm{PI} / \mathrm{Ann} \mathrm{V}^{+}$cells shown as $\%$ apoptotic cells) and by cleaved PARP by Western blot analysis and densitometry (C). Data are expressed as the mean \pm SD of three experiments. $* p$ value $<0.005$ compared to control siRNA. 
active in transformed cells. Using multiple cell types and approaches, we found that: (i) recombinant human PFKFB4 has far more kinase activity than phosphatase activity (kinase:phosphatase ratio $=4.3: 1$ ); (ii) selective inhibition of PFKFB4 using two unique siRNA molecules reduces the intracellular $\mathrm{F} 2,6 \mathrm{BP}$ in six of seven cancer cell lines indicating that endogenous PFKFB4 synthesizes F2,6BP in these cancer cells; (iii) over-expression of PFKFB4 increases the F2,6BP in all examined cancer cell lines; and (iv) genomic deletion of the $P f k f b 4$ gene reduces F2,6BP in LT-immortalized fibroblasts. Taken together, these in vitro data provide overwhelming evidence that the main biochemical function of PFKFB4 is to synthesize F2,6BP. We also found that PFKFB4 siRNA reduces glycolysis and ATP and increases the steady-state concentration of NADPH in vitro, all expected metabolic effects of reduced F2,6BP. Additionally, we observed that PFKFB4 shRNA suppressed the glucose uptake, F2,6BP,
ATP and growth of H460 xenograft tumors in mice, thus providing further evidence that the main function of PFKFB4 in vivo is to synthesize F2,6BP and thus activate PFK-1 and glycolysis. The rescue of transformed cells from PFKFB4 inhibition with the addition of pyruvate, which provides substrate for mitochondrial ATP production, provides further support that PFKFB4 is predominantly functioning to stimulate ATP production. To summarize, these data indicate that the kinase activity and, thus, the capacity to produce $\mathrm{F} 2,6 \mathrm{BP}$, serves as the dominant function of PFKFB4 in cancer cells.

Since the majority of human cancers display elevated glucose uptake and several targeted agents such as the BRAF inhibitor, vemurafenib, and the estrogen antagonist, fulvestrant, acutely suppress glucose metabolism in order to inhibit tumor growth [17, 27], we believe that PFKFB3 and PFKFB4 kinase inhibitors may prove effective for the treatment of cancer. Importantly,

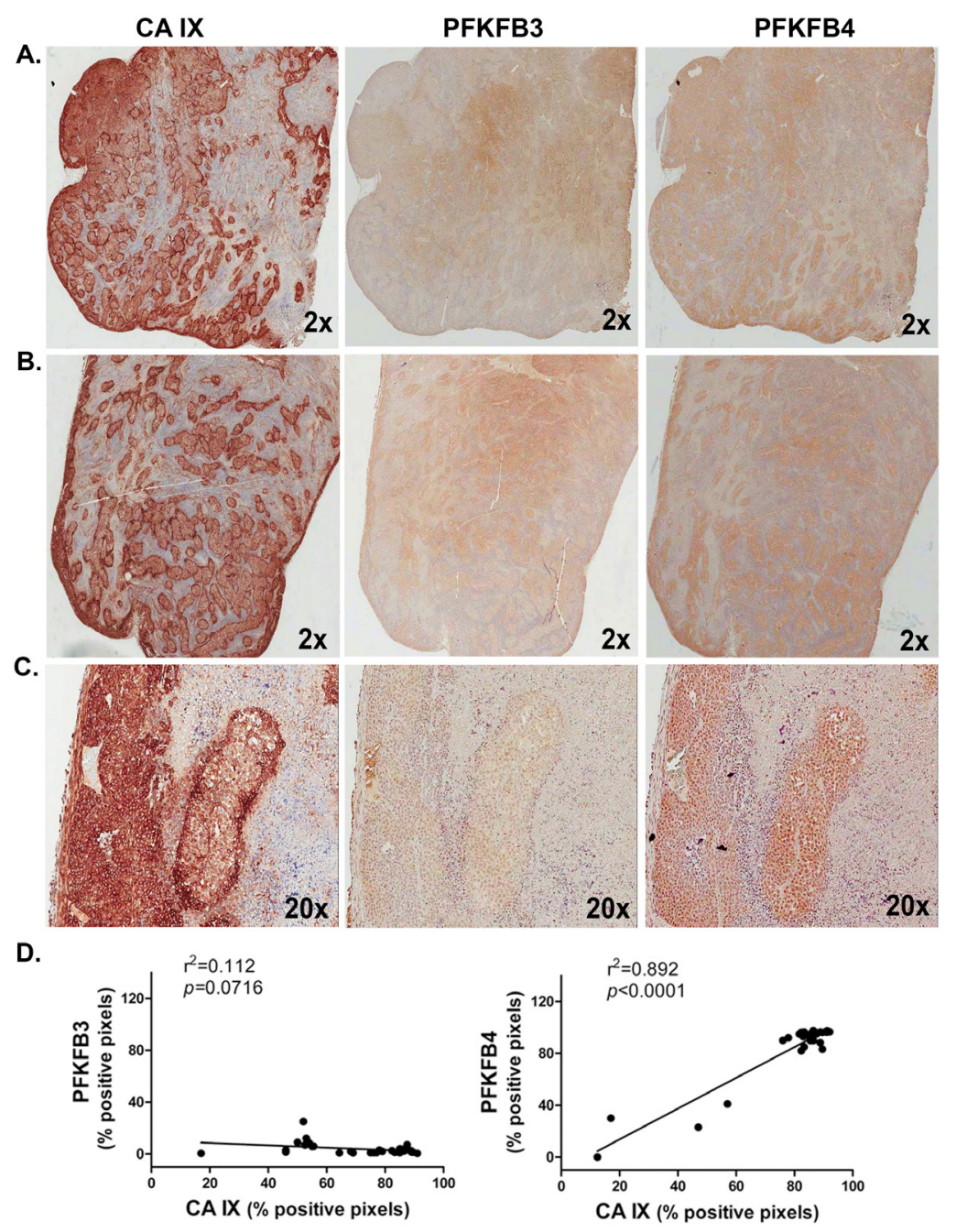

Figure 9: PFKFB4 Expression Correlates with Hypoxia In Human Lung Adenocarcinoma Xenografts. Ten human H460 lung adenocarcinoma xenografts were analyzed by immunohistochemistry for carbonic anhydrase (CA) IX, PFKFB3 and PFKFB4. Representative adjacent sections from two tumors are provided (A,B). 20X magnification of portion of tumor in (B) shown (C). Positive pixels were enumerated in a minimum of 5 fields per tumor section followed by linear regression analysis (D). $\mathrm{r}^{2}$ and $p$ values are provided. 
a novel family of related small molecule antagonists of the kinase domain of PFKFB3 (i.e. 3PO, PFK15 and PFK158) have been found to reduce glycolytic flux, ATP and cancer cell viability $[17,21,28]$. PFK158 is now being tested in a phase I clinical trial of advanced cancer patients (clinicaltrials.gov \#NCT02044861) and recently has been found to synergistically interact with targeted cancer agents [17]. Given that PFKFB3 and PFKFB4 are co-expressed in several cancer cell lines and have the redundant function of synthesizing F2,6BP, we postulate that selective inhibition of a single family member may result in compensation by a co-expressed PFKFB family member, and that combinations of PFKFB3 and PFKFB4 inhibitors may be necessary in order to fully suppress tumor glucose metabolism and growth. Future studies will be directed at creating double $P f k f b 3$ and $P f k f b 4$ knockout mice in order to conduct in vivo studies of the roles of these two seemingly redundant enzymes in regulating the glucose metabolism and growth of tumors.

Although this is the first comprehensive analysis of the relative importance of the kinase activity of PFKFB4 in transformed cells to include a measurement of human recombinant protein activity and a combination of siRNA knock-down, plasmid-based over-expression and genomic deletion experiments in multiple cancer cell types, two prior studies previously have supported the conclusion that the kinase domain of PFKFB4 was essential for cancer cell proliferation. A PFKFB4 siRNA was found to reduce F2,6BP and glycolytic flux to lactate in A549 lung adenocarcinoma cells (U.S. Patent \#8,283,332) and two other PFKFB4 siRNAs were independently observed to reduce lactate secretion and the intracellular ATP in malignant glioma cells [14]. However, a third study found that PFKFB4 siRNA increased the F2,6BP concentration of three prostate cancer cell lines [15] including the LNCaP and PC3 cell lines (using one of the two siRNAs examined in the current study). Although it is difficult to reconcile the findings from this single discordant report with those of the other two studies as well as the present study, we believe that the combined use of recombinant protein analyses, cell lines derived from several types of cancer, two methods to inhibit PFKFB4 (siRNA and genomic deletion) and PFKFB4 over-expression experiments provide comprehensive and compelling data demonstrating that the kinase activity of PFKFB4 dictates the F2,6BP concentration in transformed cells. Whereas we look forward to continued independent examinations of the biochemical functions of PFKFB4 in transformed cells, our current data support an essential role of the PFKFB4 kinase activity for cancer cell survival as well as the development of small molecule antagonists that target the kinase domain of PFKFB4.

Although the differences in metabolic functions of PFKFB3 and PFKFB4 are poorly understood, we observed a more robust induction of PFKFB4 protein relative upon exposure of $\mathrm{H} 460$ cells to $1 \%$ oxygen and significant correlation between hypoxia and PFKFB4 expression in lung adenocarcinoma tissues. Additionally, we detected a greater reduction in glycolytic flux to glutamate and lactate caused by PFKFB4 siRNA relative to PFKFB3 siRNA transfection. Given these results, we suspect that PFKFB4 may serve as a unique regulator of the glycolytic response to hypoxia. This hypothesis is supported by the observation that the H460 cells underwent increased hypoxia-associated apoptosis when subjected to PFKFB4 siRNA transfection but not when subjected to PFKFB3 siRNA transfection. These data suggest that tumors that are especially affected by poor vasculature and hypoxia may be most responsive to PFKFB4 inhibition.

\section{CONCLUSIONS}

In conclusion, our data indicate that the PFKFB4 family member functions predominantly to synthesize $\mathrm{F} 2,6 \mathrm{BP}$ which, in turn, is required for glycolytic flux through PFK-1 and subsequent ATP production. This conclusion is consistent with the finding that H460 cells are exquisitely sensitive to PFKFB4 inhibition under hypoxic conditions when the efficiency of ATP production by the mitochondria is compromised and the reliance on glycolysis to produce ATP is greatest. With the key intramolecular target of PFKFB4 now established, we believe that PFKFB4 inhibitors can be rationally designed and that these agents may prove most useful when combined with PFKFB3 inhibitors in order to circumvent potential compensation between these two PFKFB family members.

\section{METHODS}

\section{Cell lines and cell culture}

A549 and H460 NSCLC, MCF7 breast adenocarcinoma, LNCaP, PC3 and DU145 prostatic adenocarcinoma and HCT116 colon adenocarcinoma cell lines were obtained from ATCC (Manassas, VA). $\mathrm{PFKFB}^{-/}$ear pinna fibroblasts were isolated from TamCre/loxP/PFKFB4 ${ }^{-/}$mice and immortalized by transduction with REBNA/IRES retrovirus expressing SV40 large $\mathrm{T}$ antigen as described previously [20]. Cell lines were grown in DMEM (A549, LNCaP, PC3, DU145 and PFKFB44 ${ }^{-/}$), RPMI 1640 (H460), McCoy's 5A media (HCT116) or improved MEM (MCF7) (all from Invitrogen, Grand Island, NY) containing $10 \%$ fetal calf serum (FCS, Hyclone, Logan, UT) at $37^{\circ} \mathrm{C}$ in $5 \% \mathrm{CO} 2$. In some experiments, 4-hydroxytamoxifen (4OHT, 4HT, Sigma -Aldrich, St. Louis, MO) was added to PFKFB4 - fibroblasts at indicated concentrations. 


\section{Transfections}

For siRNA experiments, cells growing in 6-well plates were transfected with control siRNA (Stealth Negative Control Medium GC Duplex, Invitrogen), PFKFB3 siRNA (FB3; HSS107860, Invitrogen) or PFKFB4 siRNA (FB4 ${ }^{1}$, FB4; HSS107863, Invitrogen and FB42; siGENOME human PFKFB4 5210 \#2, Dharmacon) using Lipofectamine RNAiMax (Invitrogen, Carlsbad, CA), and harvested 48 hours after transfection. For hypoxia experiments, cells were transfected with siRNA and, after 24 hours, were placed in a hypoxia chamber (Billups-Rothenburg, Del Mar, CA) purged with 1\% oxygen for 24 hours. For short hairpin RNA (shRNA) experiments, sense and antisense DNA oligonucleotides for PFKFB4 were designed with a hairpin against the target sequences 5'-cacttgtatggtcctgt-3' and 5 '-ggagagcgaccatcttt-3' were produced by IDT (Coralville, IA). The oligonucleotides were annealed and ligated into pSUPER.neo vector (OligoEngine, Seattle, WA) following manufacturer's instructions. H460 cells were transfected with an shRNA-expressing plasmid targeted against PFKFB4 (shFB4) or a scrambled shRNA expressing plasmid (Control) using Lipofectamine 2000 (Invitrogen, Carlsbad, CA) and clones selected with G418 (500 $\mu \mathrm{g} / \mathrm{ml})$. For overexpression experiments, cells were transfected with pCMV-XL4 (vector) or pCMV-XL4 containing full-length PFKFB4 (Origene, Rockville, MD) using Lipofectamine 2000 and harvested after 48 hours.

\section{PCR analyses}

Multiplex mRNA primers were custom synthesized (IDT) against human PFKFB1-4 as described previously [20]. cDNA from normal human tissues and matched tumor and adjacent normal tissues (Clontech, Mountain View, CA) were analyzed using these primers and standard PCR conditions. PFKFB1-4 mRNA expression was determined using real-time RT-PCR with TaqMan probes for human PFKFB1-4 and $\beta$-actin (Applied Biosystems, Foster City, CA) in triplicate in 96-well optical plates (MicroAMP®, Applied Biosystems). Analysis of results and fold differences between samples were determined using StepOne software (version2.1) (Applied Biosystems) and calculated from the $\Delta \Delta \mathrm{CT}$ values with the formula $\left(2^{-\triangle \Delta C T}\right)$. The data are represented as the mean \pm SD from triplicate measurements from three independent experiments. For calculation of copy number, the molecular weight was determined for the double stranded DNA sequences of the 4 PFKFB isoforms. The OD of the DNA (in $\mathrm{gm} / \mu \mathrm{L}$ ) was divided by the molecular weight of the product (result in moles/ $\mu \mathrm{L}$ ) and then this number was multiplied by Avogadro's number $\left(6.022 \times 10^{23}\right.$ molecules/ mole). The resulting number of DNA molecules per $\mu \mathrm{L}$ was used to generate a standard curve from which copy numbers were calculated. Statistical significance was assessed by the two-sample $t$ test (independent variable).

\section{Protein extraction and Western blotting}

Cells were harvested, washed X1 in PBS and lysed in $1 X$ lysis buffer (Pierce Biotechnology, Rockford, IL) containing protease inhibitors. Protein samples were resolved on 4-20\% SDS-PAGE gels (BioRad, Hercules, $\mathrm{CA}$ ) and transferred to PVDF membranes (BioRad). After blocking in TBS containing $0.1 \%$ Tween 20 (TBS-T) and $5 \%$ milk, membranes were probed with antibodies to PFKFB3 (Proteintech, Chicago, IL), PFKFB4 (Abcam, Cambridge, MA) or $\beta$-actin (Sigma, St. Louis, MO). Secondary antibodies used were HRP-conjugated goat anti-rabbit or anti-mouse (1:5000, Pierce Biotechnology). Scanned images were quantified by densitometric analyses using Image J software (http://rsb.info.nih.gov/ij/). Values obtained were normalized to $\beta$-actin and expressed in densitometric units as a percentage of control. The data represented are the mean $\pm \mathrm{SD}$ from triplicate measurements from three independent experiments. Statistical significance was assessed by the two-sample $t$ test (independent variable).

\section{Kinase and Bisphosphatase assays}

Fructose-6-phosphate kinase and fructose-2,6bisphosphatase activities of human recombinant PFKFB3 and PFKFB4 were assayed using previously described methods wherein one unit of activity was defined as the amount of enzyme that catalyzes the formation of $1 \mu \mathrm{mol}$ of F2,6BP per min. [7, 8]. Aliquots of the reaction mixture were removed at intervals, added to $0.1 \mathrm{~N} \mathrm{NaOH}$ and then neutralized to a pH of 7.2 and assayed for F2,6BP as described below.

\section{F2,6BP measurements}

Cells were harvested, washed twice with PBS, lysed in $\mathrm{NaOH} /$ Tris acetate by heating at $80^{\circ} \mathrm{C}$ for $5 \mathrm{~min}$. Lysates were neutralized to $\mathrm{pH} 7.2$ with ice-cold acetic acid and HEPES. F2,6BP content was measured using a coupled enzyme reaction following the method of Van Schaftingen et al [29]. The F2,6BP concentration was normalized to total cellular protein measured by the bicinchoninic acid assay (BCA, Thermo Scientific, Rockford, IL). All data are expressed as the mean \pm SD of three experiments. Statistical significance was assessed by the two-sample $t$ test (independent variable). 


\section{Glycolysis assay}

Cells growing in 6-well plates were incubated in $500 \mu \mathrm{l}$ of complete medium containing $1 \mu \mathrm{Ci}$ of $5-\left[{ }^{3} \mathrm{H}\right]$ glucose per well for $60 \mathrm{~min}$ in $5 \% \mathrm{CO}_{2}$ at $37^{\circ} \mathrm{C}$. The medium was then collected and centrifuged for $5 \mathrm{~min}$ at $8000 \mathrm{rpm}$ to pellet any suspended cells. To separate the ${ }^{3} \mathrm{H}_{2} \mathrm{O}$ formed via glycolysis from the $5-\left[{ }^{3} \mathrm{H}\right]$ glucose added to the medium, an evaporation technique in a sealed system was utilized. Briefly, $150 \mu \mathrm{l}$ aliquots of medium were added to open tubes that were placed upright inside scintillation vials containing $1 \mathrm{ml}$ of $\mathrm{H}_{2} \mathrm{O}$. The scintillation vials were sealed, and the ${ }^{3} \mathrm{H}_{2} \mathrm{O}$ produced by glycolysis through enolase and released to the medium was allowed to equilibrate with the $\mathrm{H}_{2} \mathrm{O}$ in the outer vial for $48 \mathrm{~h}$ at $37^{\circ} \mathrm{C}$. The amounts of ${ }^{3} \mathrm{H}_{2} \mathrm{O}$ that had diffused into the surrounding $\mathrm{H}_{2} \mathrm{O}$ was measured on a Tri-Carb 2910 liquid scintillation analyzer (Perkin Elmer, Boston, MA) and compared with ${ }^{3} \mathrm{H}_{2} \mathrm{O}$ and $5-\left[{ }^{3} \mathrm{H}\right]$ glucose standards. Protein concentration was determined using the BCA assay and counts were normalized to protein concentration. All data are expressed as the mean $\pm \mathrm{SD}$ of three experiments. Statistical significance was assessed by the two-sample $t$ test (independent variable).

\section{2-[1-14C]-Deoxy-D-Glucose Uptake}

Cells were placed in glucose-free media for 30 minutes, $2-\left[1-{ }^{14} \mathrm{C}\right]$-deoxy-D-glucose $(0.25 \mu \mathrm{Ci} / \mathrm{mL}$; Perkin Elmer) was added for an additional $60 \mathrm{~min}$ and the cells then were washed thrice with ice-cold glucosefree media. Cell lysates were collected in $500 \mu \mathrm{L}$ of $0.1 \%$ SDS, and scintillation counts (counts $/ \mathrm{min}$ ) were measured on $400 \mu \mathrm{L}$ of the lysate. Counts were normalized to protein concentration measured by the BCA assay and data are represented as mean $\pm \mathrm{SD}$ from triplicate measurements from three independent experiments. Statistical significance was assessed by the two-sample $t$ test (independent variable).

\section{ATP Measurements}

Cell pellets were lysed using Passive Lysis buffer (1X, Molecular Probes, Invitrogen). Lysates were flash frozen in liquid nitrogen and thawed (to $37^{\circ} \mathrm{C}$ ) once to accomplish complete lysis and then centrifuged at $4^{\circ} \mathrm{C}$ for 30 seconds to clear the lysates. Intracellular ATP levels were determined using a bioluminescence assay (Molecular Probes, Eugene, OR) utilizing recombinant firefly luciferase and its substrate, d-luciferin and following manufacturer's instructions. The luminescence was read in a TD-20/20 luminometer (Turner Designs, Sunnyvale, CA) at $560 \mathrm{~nm}$. The ATP values were calculated using an ATP standard curve. The protein concentrations of the lysates were estimated using the BCA assay and ATP was expressed as pmol per $\mu \mathrm{g}$ protein. All data are expressed as the mean \pm SD of three experiments. Statistical significance was assessed by the two-sample $t$ test (independent variable).

\section{NADPH Measurements}

Cells were harvested, washed with ice cold PBS and homogenized in NADPH extraction buffer (EnzyFluo, Bioassay Systems, Hayward, CA). NADPH was then measured following manufacturer's instructions (Bioassay Systems) by reduction of the provided probe into a fluorescent product measured at $\lambda_{\text {ex/em }}$ of $530 / 585$ $\mathrm{nm}$. Values were compared with a standard curve. All data are expressed as the mean $\pm \mathrm{SD}$ of three experiments. Statistical significance was assessed by the two-sample $t$ test (independent variable).

\section{Apoptosis Assay}

Cells were stained with FITC-labeled annexin-V and propidium iodide following the manufacturer's protocol (BD Biosciences, San Diego, CA). Briefly, cells were detached and 100,000 cells per sample were pelleted by centrifugation at $1500 \mathrm{rpm}$ for 5 minutes and washed X1 with PBS. The cells were then resuspended in $1 \mathrm{X}$ binding buffer, annexin-V/FITC and/or propidium iodide added and were incubated in the dark at room temperature for 15 minutes. $1 \mathrm{X}$ binding buffer was added to increase volume and 10,000 events were counted for each sample using the appropriate filters for FITC and PI detection (BD FACSCalibur, San Jose, CA). Data was analyzed using FlowJo software (TREE STAR Inc, San Carlos, $\mathrm{CA})$. Results were calculated as the average of triplicate samples $\pm \mathrm{SD}$.

\section{NMR Experiments}

Cells treated with siRNA were grown in media containing uniformly labeled ${ }^{13} \mathrm{C}$-glucose $(2 \mathrm{gm} / \mathrm{L}$, Cambridge Isotopes Laboratories, Andover, MA) for 24 hours. Media samples were frozen in liquid nitrogen. Cells were counted and equal numbers of cells were pelleted, washed twice with cold PBS to remove adhering medium, and flash frozen in liquid nitrogen. The cell pellets and media samples were extracted with $10 \%$ icecold trichloracetic acid (TCA) followed by lyophilization. The extracts were redissolved in $\mathrm{D}_{2} \mathrm{O}$ containing 85 $\mu \mathrm{M}$ (media) or $95 \mu \mathrm{M}$ (cells) DSS (2, 2-dimethyl-2silapentane-5-sulfonate sodium salt) as both a chemical shift reference and as a concentration standard and loaded into $5 \mathrm{~mm}$ Shigemi tubes (Shigemi, Tokyo, Japan). Nuclear magnetic resonance (NMR) spectra were recorded 
at $14.1 \mathrm{~T}$ on a Varian Inova spectrometer at $20^{\circ} \mathrm{C}$ using a $90^{\circ}$ excitation pulse as described previously [30]. 1D proton NMR spectra of extracts were recorded with a 2 sec acquisition time and a total recycle time of $5 \mathrm{sec}$, with presaturation of the residual HOD resonance for 3 seconds. For analyzing the cellular extracts and determining the positional enrichment with ${ }^{13} \mathrm{C}$ we used $2 \mathrm{D}$ experiments (TOCSY and ${ }^{1} \mathrm{H}\left\{{ }^{13} \mathrm{C}\right\}$-HSQC), and quantified the ${ }^{13} \mathrm{C}$ satellite and ${ }^{12} \mathrm{C}$ peaks by volume integration in the TOCSY using established assignment protocols [30, 31]. The NMR experiments were carried out in the Structural Biology Program's NMR Core at the University of Louisville James Graham Brown Cancer Center.

\section{Mouse Studies}

H460 cells stably transfected with either PFKFB4 or control shRNA were collected from exponential growth phase culture. Cells were washed twice, re-suspended in PBS $\left(5 \times 10^{7}\right.$ cells $\left./ \mathrm{ml}\right)$ and groups of $10 \mathrm{BALB} / \mathrm{c}$ athymic female mice were injected s.c. with $100 \mu \mathrm{L}\left(5 \times 10^{6}\right.$ cells $)$ of the cell suspension. The tumors were followed from the time of appearance until the end of the experiment. Tumor masses were determined in a blinded fashion with Vernier calipers according to the following formula: weight $(\mathrm{mg})=(\text { width }, \mathrm{mm})^{2} \times($ length, $\mathrm{mm}) / 2$ [32]. All data are expressed as the mean $\pm \mathrm{SD}$ of two experiments. Statistical significance was assessed by the two-sample $t$ test (independent variable). At the end of the experiment, the animals were euthanized and tumors removed and fixed in 10\% formaldehyde. Sections were stained with hematoxylin/eosin and for immunohistochemistry as described below. At the end of the experiment, subsets of xenograft-bearing mice $(n=4)$ were injected i.p. with $2-\left[{ }^{18} \mathrm{~F}\right]$-fluoro-2-deoxyglucose $\left({ }^{18} \mathrm{~F}-\mathrm{FDG}, 150 \mu \mathrm{Ci}, 100\right.$ $\mu \mathrm{L}$ in $\mathrm{H}_{2} \mathrm{O}$ ) and, after $45 \mathrm{~min}$, were anesthetized with $2 \%$ isoflurane in oxygen and transferred to a R-4 Rodent Scanner (CTI Concorde Microsystems) micro-positron emission tomograph to capture images. Regions of interest in the tumors and cerebellum were quantified in quadruplicate and are expressed as the mean $\pm \mathrm{SD}$ of the ratio of tumor:cerebellar FDG uptake. Animal experiments were carried out in accordance with established practices as described in the National Institutes of Health Guide for Care and Use of Laboratory Animals and were approved by the University of Louisville Institutional Animal Care and Use Committee.

\section{Immunohistochemistry}

Five $\mu \mathrm{m}$ mounted sections of formalin-fixed and paraffin-embedded tumor tissue were deparaffinized with xylene. Epitope retrieval was carried out using citrate buffer in a 2100 Retriever (PickCell Laboratories). The sections were blocked with $10 \%$ goat serum for 1 hour, then incubated with primary antibody against PFKFB3, PFKFB4, carbonic anhydrase IX (Proteintech) or cleaved caspase-3 (Cell Signaling, Danvers, MA) overnight, followed by an HRP-linked goat anti-rabbit secondary antibody (1:300, Pierce Biotechnology). The sections were developed with 3,3'-diaminobenzidine tetrahydrochloride (DAB, Vector Laboratories, Burlingame, CA) for $2 \mathrm{~min}$, nuclei counterstained with Mayer's hematoxylin (SigmaAldrich) for $2 \mathrm{~min}$ and coverslips attached with Permount (Fisher Scientific, Fair Lawn, NJ). Slides were scanned using a ScanScope XT Digital Slide Scanner (Aperio), data analyzed with the positive pixel count algorithm (ImageScope, Aperio) and a minimum of 5 fields $(20 \mathrm{x}$ magnification) were quantified for each tumor section. The data is depicted as \% positive pixels/total pixels \pm SD.

\section{ACKNOWLEDGEMENTS}

We thank the following funding agencies for their support of these studies: ST, NCI 1R01CA140991 and American Cancer Society RSG-10-021-01-CNE; and JC, NCI 1R01CA149438 and NCRR CoBRE 1P30GM106396. We also thank Drs. John Eaton and Otto Grubraw for assisting with the interpretation of our data and Drs. Brian Clem and Robert Mitchell for useful discussions.

\section{Editorial note:}

This paper has been accepted based in part on peerreview conducted by another journal and the authors' response and revisions as well as expedited peer-review in Oncotarget.

\section{REFERENCES}

1. Yalcin A, Telang S, Clem B and Chesney J. Regulation of glucose metabolism by 6-phosphofructo-2-kinase/fructose2,6-bisphosphatases in cancer. Exp Mol Pathol. 2009; 86(3):174-179.

2. Okar DA, Manzano A, Navarro-Sabate A, Riera L, Bartrons $\mathrm{R}$ and Lange AJ. PFK-2/FBPase-2: maker and breaker of the essential biofactor fructose-2,6-bisphosphate. Trends Biochem Sci. 2001; 26(1):30-35.

3. Van Schaftingen E, Hue L and Hers HG. Fructose 2,6-bisphosphate, the probably structure of the glucoseand glucagon-sensitive stimulator of phosphofructokinase. Biochem J. 1980; 192(3):897-901.

4. Van Schaftingen E, Hue L and Hers HG. Control of the fructose-6-phosphate/fructose 1,6-bisphosphate cycle in isolated hepatocytes by glucose and glucagon. Role of a low-molecular-weight stimulator of phosphofructokinase. Biochem J. 1980; 192(3):887-895.

5. el-Maghrabi MR, Correia JJ, Heil PJ, Pate TM, Cobb CE 
and Pilkis SJ. Tissue distribution, immunoreactivity, and physical properties of 6-phosphofructo-2-kinase/fructose2,6-bisphosphatase. Proc Natl Acad Sci U S A. 1986; 83(14):5005-5009.

6. Manzano A, Perez JX, Nadal M, Estivill X, Lange A and Bartrons R. Cloning, expression and chromosomal localization of a human testis 6-phosphofructo-2-kinase/ fructose-2,6-bisphosphatase gene. Gene. 1999; 229(1-2):8389.

7. Sakata J, Abe Y and Uyeda K. Molecular cloning of the DNA and expression and characterization of rat testes fructose-6-phosphate,2-kinase:fructose-2,6-bisphosphatase. J Biol Chem. 1991; 266(24):15764-15770.

8. Sakakibara R, Kato M, Okamura N, Nakagawa T, Komada Y, Tominaga N, Shimojo M and Fukasawa M. Characterization of a human placental fructose-6-phosphate, 2-kinase/fructose-2,6-bisphosphatase. J Biochem. 1997; 122(1):122-128.

9. Sakakibara R, Uemura M, Hirata T, Okamura N and Kato M. Human placental fructose-6-phosphate,2-kinase/ fructose-2,6-bisphosphatase: its isozymic form, expression and characterization. Biosci Biotechnol Biochem. 1997; 61(11):1949-1952.

10. Minchenko OH, Opentanova IL, Ogura T, Minchenko DO, Komisarenko SV, Caro J and Esumi H. Expression and hypoxia-responsiveness of 6-phosphofructo-2-kinase/ fructose-2,6-bisphosphatase 4 in mammary gland malignant cell lines. Acta Biochim Pol. 2005; 52(4):881-888.

11. Minchenko OH, Ochiai A, Opentanova IL, Ogura T, Minchenko DO, Caro J, Komisarenko SV and Esumi H. Overexpression of 6-phosphofructo-2-kinase/fructose-2,6bisphosphatase-4 in the human breast and colon malignant tumors. Biochimie. 2005; 87(11):1005-1010.

12. Minchenko OH, Ogura T, Opentanova IL, Minchenko DO, Ochiai A, Caro J, Komisarenko SV and Esumi H. 6-Phosphofructo-2-kinase/fructose-2,6-bisphosphatase gene family overexpression in human lung tumor. Ukr Biokhim Zh. 2005; 77(6):46-50.

13. Minchenko O, Opentanova I and Caro J. Hypoxic regulation of the 6-phosphofructo-2-kinase/fructose-2,6bisphosphatase gene family (PFKFB-1-4) expression in vivo. FEBS letters. 2003; 554(3):264-270.

14. Goidts V, Bageritz J, Puccio L, Nakata S, Zapatka M, Barbus S, Toedt G, Campos B, Korshunov A, Momma S, Van Schaftingen E, Reifenberger G, Herold-Mende C, Lichter P and Radlwimmer B. RNAi screening in glioma stem-like cells identifies PFKFB4 as a key molecule important for cancer cell survival. Oncogene. 2012; 31(27):3235-3243.

15. Ros S, Santos CR, Moco S, Baenke F, Kelly G, Howell $\mathrm{M}$, Zamboni $\mathrm{N}$ and Schulze A. Functional metabolic screen identifies 6-phosphofructo-2-kinase/fructose-2,6biphosphatase 4 as an important regulator of prostate cancer cell survival. Cancer Discov. 2012; 2(4):328-343.
16. Cohen JS, Lyon RC, Chen C, Faustino PJ, Batist G, Shoemaker M, Rubalcaba E and Cowan KH. Differences in phosphate metabolite levels in drug-sensitive and -resistant human breast cancer cell lines determined by $31 \mathrm{P}$ magnetic resonance spectroscopy. Cancer Res. 1986; 46(8):40874090.

17. Imbert-Fernandez Y, Clem BF, O’Neal J, Kerr DA, Spaulding R, Lanceta L, Clem AL, Telang $\mathrm{S}$ and Chesney J. Estradiol stimulates glucose metabolism via 6-phosphofructo-2-kinase (PFKFB3). J Biol Chem. 2014; 289(13):9440-9448.

18. Chesney J, Mitchell R, Benigni F, Bacher M, Spiegel L, Al-Abed Y, Han JH, Metz C and Bucala R. An inducible gene product for 6-phosphofructo-2-kinase with an AUrich instability element: role in tumor cell glycolysis and the Warburg effect. Proc Natl Acad Sci U S A. 1999; 96(6):3047-3052.

19. Navarro-Sabate A, Manzano A, Riera L, Rosa JL, Ventura F and Bartrons R. The human ubiquitous 6-phosphofructo2-kinase/fructose-2,6-bisphosphatase gene (PFKFB3): promoter characterization and genomic structure. Gene. 2001; 264(1):131-138.

20. Telang S, Yalcin A, Clem AL, Bucala R, Lane AN, Eaton JW and Chesney J. Ras transformation requires metabolic control by 6-phosphofructo-2-kinase. Oncogene. 2006; 25(55):7225-7234.

21. Clem B, Telang S, Clem A, Yalcin A, Meier J, Simmons A, Rasku MA, Arumugam S, Dean WL, Eaton J, Lane A, Trent JO and Chesney J. Small-molecule inhibition of 6-phosphofructo-2-kinase activity suppresses glycolytic flux and tumor growth. Mol Cancer Ther. 2008; 7(1):110120.

22. Bobarykina AY, Minchenko DO, Opentanova IL, Moenner $\mathrm{M}$, Caro J, Esumi $\mathrm{H}$ and Minchenko $\mathrm{OH}$. Hypoxic regulation of PFKFB-3 and PFKFB-4 gene expression in gastric and pancreatic cancer cell lines and expression of PFKFB genes in gastric cancers. Acta Biochim Pol. 2006; 53(4):789-799.

23. Minchenko A, Leshchinsky I, Opentanova I, Sang N, Srinivas V, Armstead V and Caro J. Hypoxia-inducible factor-1-mediated expression of the 6-phosphofructo-2kinase/fructose-2,6-bisphosphatase-3 (PFKFB3) gene. Its possible role in the Warburg effect. J Biol Chem. 2002; 277(8):6183-6187.

24. Minchenko O, Opentanova I, Minchenko D, Ogura $\mathrm{T}$ and Esumi H. Hypoxia induces transcription of 6-phosphofructo-2-kinase/fructose-2,6-biphosphatase-4 gene via hypoxia-inducible factor-1alpha activation. FEBS Lett. 2004; 576(1-2):14-20.

25. Cordero-Espinoza L and Hagen T. Increased Concentrations of Fructose 2,6-Bisphosphate Contribute to the Warburg Effect in Phosphatase and Tensin Homolog (PTEN)deficient Cells. J Biol Chem. 288(50):36020-36028.

26. Garcia-Cao I, Song MS, Hobbs RM, Laurent G, Giorgi C, de Boer VC, Anastasiou D, Ito K, Sasaki AT, Rameh 
L, Carracedo A, Vander Heiden MG, Cantley LC, Pinton P, Haigis MC and Pandolfi PP. Systemic elevation of PTEN induces a tumor-suppressive metabolic state. Cell. 149(1):49-62.

27. McArthur GA, Puzanov I, Amaravadi R, Ribas A, Chapman P, Kim KB, Sosman JA, Lee RJ, Nolop K, Flaherty KT, Callahan J and Hicks RJ. Marked, homogeneous, and early [18F]fluorodeoxyglucose-positron emission tomography responses to vemurafenib in BRAF-mutant advanced melanoma. J Clin Oncol. 2012; 30(14):1628-1634.

28. Clem BF, O’Neal J, Tapolsky G, Clem AL, ImbertFernandez Y, Kerr DA, 2nd, Klarer AC, Redman R, Miller DM, Trent JO, Telang S and Chesney J. Targeting 6-phosphofructo-2-kinase (PFKFB3) as a therapeutic strategy against cancer. Mol Cancer Ther. 2013; 12(8):1461-1470.

29. Van Schaftingen E, Lederer B, Bartrons R and Hers HG. A kinetic study of pyrophosphate: fructose-6-phosphate phosphotransferase from potato tubers. Application to a microassay of fructose 2,6-bisphosphate. Eur J Biochem. 1982; 129(1):191-195.

30. Telang S, Lane AN, Nelson KK, Arumugam S and Chesney J. The oncoprotein H-RasV12 increases mitochondrial metabolism. Mol Cancer. 2007; 6:77.

31. Lane AN, Fan TW and Higashi RM. Isotopomer-based metabolomic analysis by NMR and mass spectrometry. Biophysical Tools for Biologists 2008; 84:541-588.

32. Taetle R, Rosen F, Abramson I, Venditti J and Howell S. Use of nude mouse xenografts as preclinical drug screens: in vivo activity of established chemotherapeutic agents against melanoma and ovarian carcinoma xenografts. Cancer Treat Rep. 1987; 71(3):297-304. 